\title{
Herausforderungen der Qualitätssicherung - Nachhaltigkeit und Verbreitung
}

\section{Christoph Röder}

Institutsleiter a.i.

MEM Forschungszentrum

Korrespondenz:

Prof. Dr. med. Christoph Röder, MPH

Institut für Evaluative Forschung in der Orthopädie

Universität Bern

Stauffacherstrasse 78

CH-3014 Bern

Tel. 0316315932

Fax 0316315960

christoph.roeder[at]

memcenter.unibe.ch
Es gibt keine allgemein akzeptierte Definition von Qualität im Gesundheitswesen, diejenige des USInstitutes für Medizin (IoM) wird jedoch häufig verwendet: das Aussmass, in dem Gesundheitsdienstleistungen für Einzelpersonen und Bevölkerungsgruppen die Wahrscheinlichkeit erhöhen, die beabsichtigten Behandlungsergebnisse zu erzielen und konsistent mit aktuellem medizinischen Wissen sind. Das IoM hat 6 Dimensionen identifiziert, durch die Qualität ausgedrückt wird: Sicherheit, Wirksamkeit, Patientenzentriertheit, Rechtzeitigkeit, Effizienz, Gerechtigkeit.

Es gibt zahlreiche spezifische Ansätze, um Qualität zu verbessern, wie etwa Lean oder Six Sigma, aber keine klare Evidenz bzgl. der Über- oder Unterlegenheit eines dieser Ansätze. Vielmehr ist die Art der Implementierung der Veränderungen von vitaler Bedeutung. Hier spielen Faktoren wie Führungskompetenz, Einbezug der klinisch tätigen Angestellten, Fokussierung und bereitgestellte Ressourcen, um die Veränderungen zu erleichtern, zentrale Rollen. Gemäss der NHS Health Foundation führen nur ca. zwei Drittel der Verbesserungsinitiativen im Gesundheitswesen zu nachhaltigen und andauernden Veränderungen, welche die beabsichtigten Ziele auch erreichen. Dementsprechend müssen die Führungsebenen der jeweiligen Institutionen darüber nachdenken, wie sie solche Veränderungen am besten in ihren Betrieben einbinden. Es gibt Hinweise in der Literatur, dass nachhaltige Veränderungen eher aus Modellen resultieren, welche Patienten und Personal bei Entwicklung, Design und Implementierung einbinden, statt aus Befehls- und kontrollbasierten Modellen.

\section{Swiss Quality Award \\ INNOVATIONS IN HEALTHCARE}

Swiss Quality Award 2014: les inscriptions sont ouvertes

Le Swiss Quality Award distingue chaque année des projets consacrés à la qualité. II offre une vitrine aux pionnières et pionniers qui font preuve de créativité et s'engagent sur des voies novatrices pour les démarches qualité. Attribué conjointement par la Fédération des médecins suisses (FMH), l'Institut de recherche évaluative en médecine (IEFM) de I'Université de Berne et la Société suisse pour le management de qualité dans la santé (SQMH), le Swiss Quality Award se décline en trois catégories distinctes: secteur ambulatoire, secteur hospitalier et projet intersectoriel; chaque catégorie étant dotée d'un prix de 10000 francs. En outre, une récompense d'une valeur de 2000 francs est décernée pour le meilleur poster.

Vous avez mis en œuvre un projet innovant en faveur de la qualité? Alors n'hésitez pas à vous inscrire! Le délai d'inscription est fixé au 30 avril 2014. Pour de plus amples informations, veuillez consulter le site www.swissqualityaward.ch.

In jedem Fall scheitern viele Verbesserungsinitiativen daran, dass sie im Rahmen des betreffenden Projektes stagnieren, ohne Wirksamkeit nach Projektabschluss zu erzielen. Nachhaltigkeit wird von Initiativverfall und Verpuffung des Verbesserungseffektes kompromittiert, was schlussendlich als eine Verschwendung von Mitteln und Ressourcen betrachtet werden muss. Zusätzlich führt der Mangel an Verbreitung von Innovationen oft $\mathrm{zu}$ «Verbesserungsinseln» oder «Exzellenzzonen». Erfolgsmodelle tendieren dazu, am Ort der Initiierung zu verbleiben, und ihre Resultate lassen sich nur im Kontext des lokalen Projektes beobachten. Mit wirksamen Verbreitungsmechanismen könnten die positiven Effekte solcher Innovationen jedoch vervielfacht werden und Verbesserungen im Gesundheitswesen beschleunigt werden.

Das diesjährige Nationale Symposium für Qualitätsmanagement im Gesundheitswesen widmet sich am 17. September in Solothurn unter dem Titel «Erfolge im Qualitätsmanagement: von Scheitern und Nachhaltigkeit» dieser Thematik.

Es würde den Rahmen dieses Artikels sprengen, Nachhaltigkeit und Verbreitung von Qualitätsverbesserung im Gesundheitswesen in ausreichender Tiefe zu behandeln. Der Autor möchte jedoch auf drei sehr pragmatisch geschriebene Quellen verweisen, aus denen hier noch einige wichtige Punkte erwähnt werden sollen.

- Quality improvement made simple. What every board should know about healthcare quality improvement. The Health Foundation (www.health. org.uk)

- Guide on spread and sustainability. Healthcare Improvement Scotland (www.healthcareimprovementscotland.org)

- The Improving Improvement Action Evaluation Project: Making temporary improvement continuous (www.skl.se, besser Google)

- AHRQ Quality Indicators Toolkit (www.ahrq.gov/ professionals/systems/hospital/qitoolkit/index.html)

10 Schritte für nachhaltige Verbesserungen im Gesundheitswesen:

1. Wähle den richtigen Ansatz, um das Problem zu identifizieren: Probleme, auf die sich das Verbesserungsprojekt bezieht, sollten bedeutend für Patienten, professionelles Personal und Management und lösbar sein. Wähle Probleme von allgemeiner Bedeutung, welche nicht zu komplex sind.

2. Minimiere Zeitkonflikte: Ermögliche dem Personal, an Qualitätsaktivitäten zu arbeiten, indem Extrapersonal angestellt wird oder weniger Patien- 
ten behandelt werden. Organisiere Training und Qualitätsaktivitäten zu geeigneten Zeiten. Stelle Daten und administrative Unterstützung zur Verfügung, und lasse das Management klare $\mathrm{Ar}$ beitsprioritäten festlegen.

3. Stelle sicher, dass das Personal Qualitätsmethodik kennt und diese richtig anwendet: durch das richtige Training, Qualitätscoaches und Berichterstattung an das Management.

4. Mache Qualität zu einer klaren Managementverantwortlichkeit und verbinde bestimmte Qualitätsaktivitäten mit der jeweiligen Führungsebene: durch Berichterstattung, Anreiz- und Bonussysteme, die Qualitätsverbesserung attraktiv machen.

5. Wähle das richtige Problem, und messe Ergebnisse und Fortschritte: Wähle ein Methodik, die es ermöglicht, organisatorisch wichtige und lösbare Probleme zu identifizieren, welche für Qualitätsverbesserungsmassnahmen geeignet sind, da sie messbare Ziel- und Progressindikatoren mit sich bringen; erfasse entsprechende Daten für deine Berichterstattung.

6. Untersuche Wirksamkeit und handle: Sammle Evidenz für die Wirksamkeit deiner Qualitätsinitiative und stelle dabei sicher, dass alle Managementebenen das Kosten-Nutzen-Verhältnis beachten. Mache erfolgreiche Aktivitäten publik. Evidenz über Wirksamkeit garantiert die Fortführung des Projektes nicht, aber sie hilft.

7. Stoppe Qualitätsinitiativen, die nicht wirksam sind: durch frühe Expertenbeurteilung, aber erst nach einer Mindestzeit, um Progressindikatoren aufgenommen und evaluiert haben zu können.

8. Schütze wirksame Projekte: von äusseren Störfaktoren wie organisatorischen Veränderungen, Personalfluktuation und unmittelbaren Krisen - andernfalls kann die Investition und die Glaubwürdigkeit des Projektes verlorengehen.

9. Formalisiere und überarbeite periodisch: Sichere die vorgenannten Aspekte ab, indem Qualitätsverantwortlichkeiten in Stellenbeschreibungen aufgenommen werden, Qualitätsindikatoren zu Leistungsbeurteilungen und Evaluationssystemen hinzugefügt werden, um formelle Anerkennung und Honorierung von Qualitätsarbeit zu etablieren, und indem Standardisierung durch Prozeduren, Prozesse und Dokumentation erreicht wird. Überarbeite diese bürokratischen Massnahmen periodisch, je nachdem wie die Betriebsbedingungen sich verändern. Institutionalisiere und integriere Qualitätsverbesserung, aber verknöchere sie nicht.

10. Entwickle eine Strategie und ein System zur Nachhaltigkeitssicherung der Qualitätsmassnahmen: Ein solches Vorhaben muss geplant und durchgeführt werden und regelmässig überarbeitet werden, wenn die Umgebungsbedingungen sich verändern. Das strategische System zur Nachhaltigkeitssicherung einer bestimmten Institution muss nicht notwendigerweise alle vorgenannten Massnahmen beinhalten.

Selbst in den kürzesten und handlichsten Leitlinien zu Qualität, Nachhaltigkeit und Verbreitung wird deutlich, dass es sich hier um komplexe, aufwendige, und niemals endende Aktivitäten handelt, die unbedingt vom obersten Management anerkannt und gefördert werden müssen, Teamarbeit, Expertise, Planung, Disziplin und Ressourcen benötigen, die betroffenen Mitarbeiter und Patienten einbeziehen müssen, und die trotzdem nicht immer zu einem messbaren oder erwünschten Resultat führen werden. Einige der wichtigsten Prinzipien sind Datenerfassung und Auswertung zur Messung des Projekt- (Implementierungsphase) und Programmwirkungsgrades (Nachhaltigkeitsphase); Einsicht in und Verständnis für die betroffenen Prozesse, um die Daten richtig interpretieren zu können; Steigerung der Zuverlässigkeit einer Verbesserungsmassnahme, um diese robuster gegen Veränderungen im Umfeld $\mathrm{zu}$ machen und um Verschwendung von Ressourcen und Systemfehler zu reduzieren, welche Gefährdung für Personal oder Patienten mit sich bringen können; Verständnis von Bedarf, Kapazität und Mengendurchflüssen im entsprechenden Bereich; Begeisterung, Einbezug und Beteiligung des Schlüsselpersonals; Einbezug von Patienten und deren Sicht des Problems und dessen potentieller Lösung.

\section{September 2014 - Landhaus Solothurn:}

\section{Nationales Symposium für Qualitätsmanagement im Gesundheitswesen}

\section{«Erfolge im Qualitätsmanagement: Von Scheitern und Nachhaltigkeit»}

Qualitätssicherung erfolgreich und nachhaltig zu machen ist eine kontinuierliche Aufgabe, und in Anbetracht der andauernden Veränderungen im Gesundheitswesen nur möglich, wenn der Ansatz von oben getragen und gestützt wird. Doch in Nachhaltigkeit und Verbreitung liegen auch ökonomische Potentiale, die als Selektions- und Positionierungsfaktoren einer Gesundheitsinstitution verwendet werden können. Zunehmend wird die Diskussion über das Gesundheitswesen der Zukunft eng mit der Auseinandersetzung um das Thema Qualität und Nachhaltigkeit geführt, wozu auch das Scheitern entsprechender Bemühungen gehören muss. Experten berichten über Ansätze, Qualitätsprojekte zu Qualitätsprogrammen zu machen, und eine Umfrage unter allen Bewerbern des Swiss
Quality Award zum Stand ihrer Projekte seit dem Zeitpunkt der Einreichung wird vorgestellt.

Im Rahmen des 7. Nationalen QM-Symposiums wird der Swiss Quality Award für herausragende Innovationen für das QM im Gesundheitswesen verliehen.

Informationen zum Programm und Anmeldeformular unter: www.qmsymposium.ch

Informationen zum Swiss Quality Award unter: www.swissqualityaward.ch 